\title{
Effect of in vitro fertilization on gene expression and development of mouse preimplantation embryos
}

\author{
Gnanaratnam Giritharan, Said Talbi, Annemarie Donjacour, Francesca Di Sebastiano, Anthony \\ T Dobson and Paolo F Rinaudo \\ Department of Obstetric and Gynecology, University of California, San Francisco, San Francisco, California 94143, \\ USA \\ Correspondence should be addressed to P F Rinaudo who is now at Division of Reproductive Endocrinology and Infertility, \\ University of California, San Francisco, 2356 Sutter Street, San Francisco, California 94115, USA; Email: rinaudop@obgyn.ucsf.edu
}

\begin{abstract}
In vitro culture (IVC) of preimplantation mouse embryos is associated with changes in gene expression. It is however, not known if the method of fertilization affects the global pattern of gene expression. We compared gene expression and development of mouse blastocysts produced by in vitro fertilization (IVF) versus blastocysts fertilized in vivo and cultured in vitro from the zygote stage (IVC) versus control blastocysts flushed out of the uterus on post coital day 3.5. The global pattern of gene expression was assessed using the Affymetrix 4302.0 chip. It appears that each method of fertilization has a unique pattern of gene expression and development. Embryos cultured in vitro had a reduction in the number of trophoblastic cells (IVF 33.5 cells, IVC 39.9 cells, and 49.6 cells in the in vivo group) and, to a lesser degree, of inner cell mass cells $(12.8,11.7$, and 13.8 respectively). The inner cell mass nuclei were larger after culture in vitro $\left(140 \mu \mathrm{m}^{2}, 113 \mu \mathrm{m}^{2}\right.$, and $86 \mu \mathrm{m}^{2}$ respectively). Although a high number of genes (1912) was statistically different in the IVF cohort when compared with the in vivo control embryos, the magnitude of the changes in gene expression were low and only a minority of genes (29 genes) was changed more than fourfold. Surprisingly, IVF embryos were different from IVC embryos (3058 genes were statistically different, but only three changed more than fourfold).

Proliferation, apoptosis, and morphogenetic pathways are the most common pathways altered after IVC. Overall, IVF and embryo culture have a profound effect on gene expression pattern and phenotype of mouse preimplantation embryos.
\end{abstract}

Reproduction (2007) 134 63-72

\section{Introduction}

It is estimated that $1 \%$ of children in the western world are born with the help of assisted reproductive techniques, in vitro fertilization (IVF), and intracytoplasmic sperm injection (ICSI). While these techniques are thought to be safe (Olivennes et al. 2002), new evidence suggests an increased risk of complications associated with their use, such as cerebral palsy and delayed development (Stromberg et al. 2002), low birth weight, increased birth defects (Anthony et al. 2002, Hansen et al. 2002, Schieve et al. 2002), and epigenetic disorders such as Angelman and Beckwith-Wiedemann Syndromes (Cox et al. 2002, DeBaun et al. 2003).

While it is possible that infertility, per se, could increase these risks (Kovalevsky et al. 2003), studies in animal models have demonstrated that in vitro culture (IVC) is suboptimal and represents an additional stress to gametes and embryos. IVC of preimplantation embryos alters their global gene expression patterns (Rinaudo \&
Schultz 2004, Rinaudo et al. 2006) and affects the behavior of mice born after such procedures (Ecker et al. 2004, Fernandez-Gonzalez et al. 2004). Unfortunately, these studies have only examined the effect of IVC on outcome and have not explored the effect of gamete manipulation by IVF or ICSI.

In order to investigate the effect that IVF has on development and expand upon our prior IVC studies (Rinaudo \& Schultz 2004, Rinaudo et al. 2006) we carried out additional experiments utilizing IVF. Specifically, morphologic and gene expression studies were performed on blastocysts fertilized in vivo but grown in vitro (IVC group) and those fertilized and grown in vitro (IVF group) when compared with in vivo controls (in vivo group). Therefore, any comparisons between the IVC and IVF groups would tease out the effects of gamete manipulation. We refer readers to our past publication for analysis of differences between IVC and in vivo embryos (Rinaudo \& Schultz 2004, Rinaudo et al. 2006). 


\section{Materials and Methods}

\section{Collection of mature oocytes and preimplantation mouse embryos}

Oocytes and embryos were isolated from super-ovulated dams as previously described (Rinaudo \& Schultz 2004). Briefly, CF-1 female mice were injected with $5 \mathrm{IU}$ pregnant mares serum gonadotrophin and $42-46 \mathrm{~h}$ later with $5 \mathrm{IU}$ human chorionic gonadotropin (hCG); some of the females were then bred to B6D2F1/J males overnight. The following morning, oocytes and zygotes were obtained from the ampullae. The zygotes were cultured in Whitten Medium (WM) to the blastocyst stage under $5 \% \mathrm{CO}_{2}$ in humidified air at $37^{\circ} \mathrm{C}$. The oocytes were incubated in WM containing $15 \mathrm{mg} / \mathrm{ml}$ BSA and sperm obtained from the cauda epididymis of male B6D2F1/J mice for $4 \mathrm{~h}$. The fertilized oocytes were washed and cultured in WM to the blastocyst stage under $5 \% \mathrm{CO}_{2}$ in humidified air at $37^{\circ} \mathrm{C}$. Only late-cavitating blastocysts of similar morphology were selected. To collect blastocysts of identical morphology, the collection had to be performed at different times: the IVF and IVC groups were harvested at $\sim 106-112 \mathrm{~h}$ post-hCG, the control in vivo group $96-100 \mathrm{~h}$ post-hCG. Each treatment was repeated four times. Each time, for the IVF group, sperm from one male was used to fertilize oocytes from two to three females. For the IVC and in vivo groups, eggs were collected from two to three females after overnight breeding (one male per female). All animal experiments were approved by the Institutional Animal Care and Use Committee.

\section{Differential embryo staining}

A modified dual nuclear staining method described by Thouas et al. (2001) was performed to differentially stain inner cell mass (ICM) and trophoblast cell nuclei of fully developed blastocysts. Briefly, the blastocysts were exposed to $1 \%$ Triton $\times 100$ (Sigma-Aldrich Co.) in minimum essential medium (MEM; Invitrogen Corporation) for 3-5 s, washed three to five times in MEM+ polyvinylpyrrolidone (PVP; Sigma-Aldrich Co.) and incubated in MEM+PVP containing $100 \mu \mathrm{g} / \mathrm{ml}$ propidium iodide (Sigma-Aldrich) for $30 \mathrm{~s}$. The embryos were then washed three to five times in MEM + PVP and fixed overnight in $100 \%$ ethanol containing $25 \mu \mathrm{g} / \mathrm{ml}$ of bizbenzamide (Sigma-Aldrich Co). The embryos were mounted on a clean glass slide using glycerol and kept in a dark chamber until observed under fluorescence light using a standard Leica microscope (Model DMRB, Leica Microsystems AG, Wetzlar, Germany).

The embryos were observed under fluorescence microscopy and the numbers of ICM (blue) and trophoblast (TB, red) nuclei were counted and photographed. The staining procedure was repeated at least five times per treatment group with different sets of blastocysts $(n \geq 45)$. The area of all the ICM cell nuclei in each blastocyst in the whole mount preparation was calculated measuring two perpendicular diameters using image processing software (Adobe Photoshop). All the ICM cell nuclei of at least 20 representative blastocysts per treatment group were measured. Margins of overlapping nuclei were carefully drawn using the color contrast of the overlapping and non-overlapping area.

\section{RNA extraction and amplification}

For every replicate total RNA was extracted from pools of 10 embryos using PicoPure RNA Isolation Kit (Arcturus, Sunnyvale, CA, USA) according to the manufacturer's instructions. A total of four replicates were performed for each experimental group. In each pool, five embryo equivalents of total RNA was used for RT followed by linear amplification of antisense cDNA strand, fragmentation, and biotin labeling by using NuGEN Ovation Biotin System (NuGEN Technologies Inc., San Carlos, CA, USA). Briefly, RNA is converted to cDNA with a unique DNA/RNA heteroduplex at one end. A linear isothermal DNA amplification process was conducted using DNA/RNA chimeric primer, DNA polymerase and RNase $\mathrm{H}$ in a homogeneous isothermal assay that provides highly efficient amplification of DNA sequences. The amplified cDNA strands were subjected to an enzymatic fragmentation and the fragmented product was then labeled with biotin. RNA and cDNA mass and size distribution were determined before and after amplification, and after fragmentation using the Agilent Bioanalyzer (Palo Alto, CA, USA).The cDNA yield before fragmentation and labeling was 12-15 $\mu$ g. Final yield of fragmented and biotinylated cDNA was 4$4.5 \mu \mathrm{g}$, of which $2.2 \mu \mathrm{g}$ were used for microarray analysis.

\section{Microarray preparation}

Fragmented and biotin labeled cDNA samples were submitted to the Genomic Core Facility of University of California San Francisco for GeneChip hybridization. The samples were hybridized to mouse Affymetrix 430 2.0 GeneChips, then washed and stained on fluidics stations and scanned at $3 \mu \mathrm{m}$ resolution according to the manufacturer's instructions (GeneChip Analysis Technical Manual, www.affymetrix.com). Four replicates per each experimental group were performed, for a total of 12 independent experiments.

\section{CDNA preparation for real-time PCR analysis}

RT was accomplished by utilizing the commercially available first strand cDNA synthesis kit (iScript cDNA Synthesis Kit, Bio-Rad Laboratories). The RT reactions were performed by following the kit manufacturer's 
protocol. For each treatment group the RT was repeated four times with different sets of blastocyst stage embryos.

The real-time PCR was performed using TaqMan Universal PCR Master Mix, Gene Expression Assays containing gene specific primers and TaqMan probe (Applied Biosystems, Foster City, CA, USA), and 0.1 embryo equivalents of cDNA. The corresponding $A B I$ TaqMan Assay-on- Demand probe/primer sets used were Mm00498012_m1 (Ube2a), Mm00438084_m1 (Ccng1), Mm00599980_m1 (Dpf1), and Mm999 99915_g1 (Gapdh). Duplicates were used for each real-time PCR; a minus template served as control. For each treatment group the real-time PCR was repeated four times. The real-time PCR data were analyzed within the log-linear phase of the amplification curve obtained for each probe/primer using the comparative threshold cycle method (Bio-Rad Laboratories).

\section{Data analysis}

The percent of embryos that developed to the two-cell stage and to the blastocyst stage and embryo cell number data were analyzed by $\chi^{2}$ and One-way ANOVA, respectively. Cell numbers were compared using a mean separation procedure when ANOVA showed significant $F$ values using Fisher's Least Significant Difference method. Results are reported as the mean values for each set of data \pm s.E.M.

\section{Microarray gene expression data analysis}

The Gene Chip arrays were first processed using GCOS Software (Affymetrix Inc., Santa Clara, CA, USA). Then input into GeneSpring version 7.2 software (Agilent Technologies, Santa Clara, CA, USA) for data analysis. The robust multiarray analysis algorithm (GeneSpring; Agilent Technologies) was applied to the data for background adjustment, normalization, and log2transformation of perfect match values (Irizarry et al. 2003). Subsequently, the data were subjected to per chip and per gene normalization using GeneSpring normalization algorithms. The resulting generated gene lists of differentially expressed genes included genes with a $P$ value of $\leq 0.05$ by a one-way ANOVA parametric test and a Benjamini-Hochberg multiple testing correction for false discovery rate.

\section{Principal component analysis (PCA)}

PCA is an unsupervised clustering method and visualization tool that is used to analyze large data sets derived from gene expression arrays analyses (Joliffe \& Morgan 1992). It reduces the variability in the data set into three dimensions representing the predominant variations in the data. Each dimension represents a component to which a certain percentage of variance in the data is attributed. The PCA algorithm was applied to all samples using GeneSpring software.

\section{Gene ontologies (GO) classification}

Differentially expressed genes among different embryo culture conditions, identified by microarray gene expression analysis, were classified into known GO using GO Tree software (http://genereg.ornl.gov/gotm/; Zhang et al. 2005). Gene ontology tree machine (GOTM) was used to interpret biological, molecular, and cellular functions of genes identified in response to different embryo culture conditions. GOTM uses GO hierarchies to derive significant biological processes, molecular functions, and cellular components in a gene list. It implements a statistical analysis of the GO categories for the input gene list and suggests biological areas of potential value for further investigation (Ashburner et al. 2000). First, the differentially expressed genes are classified by their corresponding $\mathrm{GO}$ categories, and the observed number of genes in each of these GO categories is recorded. Genes represented on the Affymetrix 4302.0 chip comprise the reference gene list. The expected number of genes in each GO category corresponds to the number of genes falling into that $\mathrm{GO}$ category in the reference gene list. A given GO category is considered enriched when the observed number of genes in that category is greater than the expected number. The GO classifications for different conditions were derived, taking into account only non-redundant and specific GO classes for each condition.

\section{KEGG pathways}

Differentially expressed genes in IVF versus control were classified in pathways. Hypergeometric testing was applied to derive the enriched genes in each pathway with a $P<0.05$ (Zhang et al. 2005). Kyoto Encyclopedia of Genes and Genomes (KEGG) pathways were derived for each culture condition as well as each K-means cluster. Only selected pathways and processes are highlighted in the results section.

\section{Results}

\section{Effect of IVF and embryo culture on embryo develop- ment and cell number}

Expanded mouse blastocysts are easily recognizable, and therefore offer an excellent endpoint for morphologic assessment. To assess developmental competence we observed how many embryos developed from the zygote stage to two-cell stage and from the two-cell stage to the blastocyst stage (Table 1). A higher number of IVF embryos developed to the blastocyst stage $(P<0.01)$ when compared with the IVC group. 
Table 1 Effect of fertilization method on preimplantation development.

\begin{tabular}{lcccccc}
\hline Treatment & Number of zygotes & $\begin{array}{c}\text { Number of two-cell } \\
\text { embryos }\end{array}$ & $\begin{array}{c}\text { Number of } \\
\text { blastocysts }\end{array}$ & $\begin{array}{c}\text { Zygote to two-cell } \\
(\%)\end{array}$ & $\begin{array}{c}\text { Two-cell to } \\
\text { blastocyst }(\%)\end{array}$ & $\begin{array}{c}\text { Zygote to blastocyst } \\
(\%)\end{array}$ \\
\hline IVF & 215 & 186 & 167 & 86.5 & $89.8^{*}$ & $77.7^{*}$ \\
IVC & 553 & 458 & 340 & 82.8 & $74.2^{+}$ & $61.4^{+}$ \\
\hline
\end{tabular}

${ }^{*}{ }^{\dagger}$ Values with different superscripts in each column differ significantly $(P<0.01)$.

In order to investigate the effect of the method of fertilization and IVC on cell division and cell allocation, we counted the number of ICM and TE cell in expanded blastocysts. It is important to notice that only late cavitating blastocysts with similar morphology as observed with a stereomicroscope were used for analysis. In vivo embryos had on average one additional ICM cell when compared with IVF embryos ( $P=0.053$, non significant) and two additional cells when compared with IVC embryos $(P<0.01)$. In addition, the area of the ICM nuclei in the IVF group was larger than the area of the ICM nuclei in the IVC group and the in vivo group $(P<0.01)$. The IVF embryos had reduced number of TE cells when compared with the IVC embryos and to the in vivo control embryos $(P<0.01$; Table 2$)$.

\section{Effect of IVF and embryo culture on global gene expression}

To analyze the global pattern of gene expression we used the new mouse Affymetrix 4302.0 microarray chip, which is believed to represent the complete mouse genome. Out of 45101 probe sets, by using a cut-off value of 50 (Baum et al. 2003) in the raw expression level, 11037 (24.4\%), 10570 (23.4\%), and 11076 $(24.5 \%)$ transcripts were considered detected in IVF, IVC, and in vivo blastocysts respectively. These results are consistent with data reported in the literature (Zeng \& Schultz 2005).

Published evidence confirms that microarray data are robust and reliable (Zeng \& Schultz 2005). Nevertheless, we conducted an additional analysis of a number of genes using real-time RT PCR to confirm the microarray results obtained in this study (Fig. 1). Results of this analysis indicated that the three genes tested (Ube2a, Ccgn1, and Tlk1) confirm the microarray changes. We then performed pairwise comparison to observe how many genes are statistically different between the three groups of embryos as demonstrated in Fig. 2. A significant number of genes are statistically misregulated following IVF (1912 transcripts) or IVC (4660 transcripts) when compared with in vivo control embryos. As expected, the magnitude of change in gene expression for most of the genes was small (Rinaudo \& Schultz $2004)$, as only $18 \%(357 / 1912)$ and $12 \%$ (555/4660) of the mis-expressed genes showed more than twofold change in IVF versus in vivo and IVC versus in vivo embryos respectively. In addition, only 27 genes from the IVF group and 35 from the IVC group genes showed more than a fourfold change. Unexpectedly, the comparison of IVC versus in vivo embryos showed a nearly double number of mis-expressed genes when compared with the IVF versus in vivo group. The direct statistical comparison of IVF versus IVC revealed that 3058 genes were differently expressed. The list of significantly different genes after each pairwise comparison with the relative expression profiles is summarized in supplemental Tables 1 (IVF versus in vivo), 2 (IVF versus IVC) and 3 (IVC versus in vivo) (which can be viewed online at www.reproduction-online.org/supplemental/).

\section{Hierarchical clustering analysis and PCA}

Hierarchical clustering analysis and PCA of the expression profiles offer the opportunity to assess how similar the different treatments are and to obtain a visual understanding of the relationship between the different experimental components (Fig. 3A and B). The results of these analyses underscore that in vivo embryos, IVC and IVF embryos have a very unique and distinctive transcriptome. Hierarchical clustering of samples using differentially expressed gene set resulted in a striking segregation of samples into two major clustering branches (Fig. 3A). All the replicate samples of in vivo control group self-cluster into one branch. Both IVF and IVC samples self-cluster into another branch that further

Table 2 Effect of fertilization method on blastocysts cell number and on the nuclear area of the ICM cells.

\begin{tabular}{|c|c|c|c|c|}
\hline Treatment & \# ICM cells (mean \pm S.E.M.) & $\begin{array}{l}\text { Area of ICM nuclei }\left(\mu \mathrm{m}^{2}\right) \\
(\text { mean } \pm \text { s.E.M. })^{\mathrm{a}}\end{array}$ & \# TE cells (mean \pm S.E.M.) & \# Total cells (mean \pm s.E.M.) \\
\hline In vivo $(n=46)$ & $13.80 \pm 0.50^{*}$ & $86.01 \pm 1.48^{\ddagger}$ & $49.67 \pm 1.50^{*}$ & $63.48 \pm 1.70^{*}$ \\
\hline IVC $(n=70)$ & $11.77 \pm 0.40^{\dagger}$ & $113.65 \pm 2.16^{+}$ & $39.99 \pm 1.22^{+}$ & $51.76 \pm 1.38^{+}$ \\
\hline $\operatorname{IVF}(n=63)$ & $12.84 \pm 0.42^{*^{+}}$ & $140.20 \pm 5.89 *$ & $33.51 \pm 1.28^{\ddagger}$ & $46.35 \pm 1.45^{\ddagger}$ \\
\hline
\end{tabular}

${ }_{*, t, \neq}$ Values with different superscripts in each column differ significantly $(P<0.01)$. At least five replicates per treatment group were performed.

${ }^{a}$ Area of the ICM nuclei is the average of all the ICM cell nuclei area (range 279-313 nuclei measured) from at least 20 representative embryos per group. $n$, number of embryos examined. 

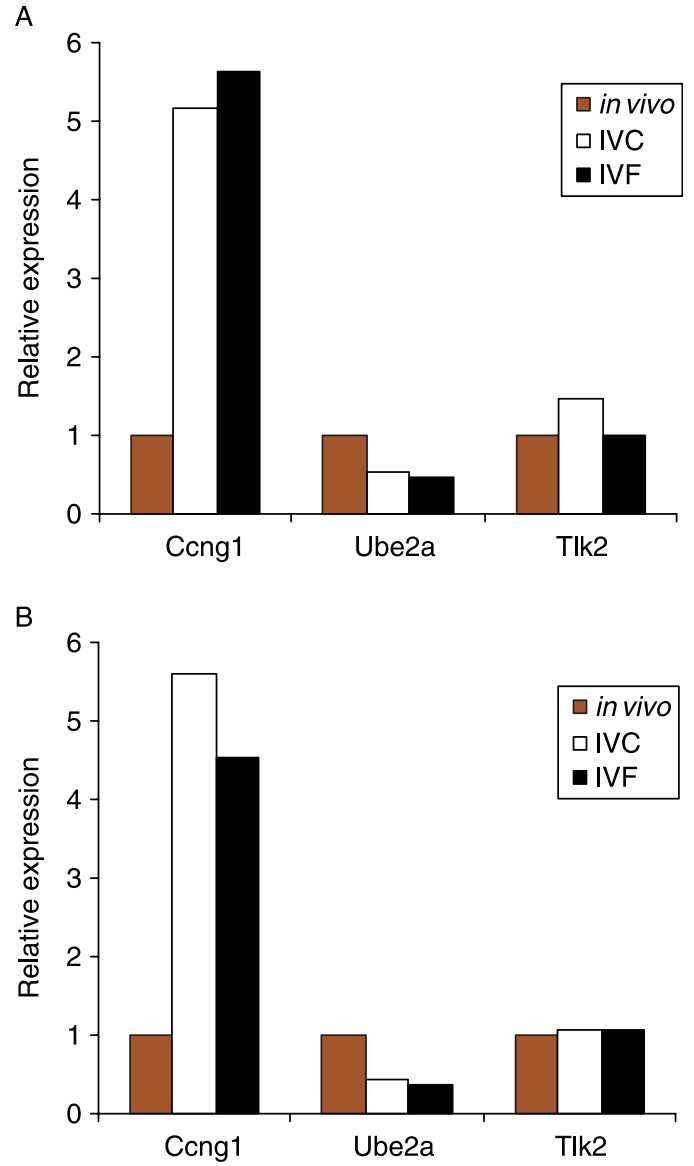

Figure 1 Real-time PCR verification of gene expression data. Expression profile of cyclin G1 (Ccng1), ubiquitin-conjugating enzyme E2A (Ube2a) and tousled-like kinase 2 ( $T / k 2$ ) genes in murine blastocysts produced by in vivo fertilization and culture (in vivo), in vivo fertilization and in vitro culture (IVC), and in vitro fertilization and culture (IVF) using both (A) microarray and (B) real-time PCR techniques.

divides into two major sub-branches and into which all the replicates from IVF and IVC self-cluster.

PCA distributes samples into a two dimensional space based on the variance in gene expressions, and samples that have similar trends in their gene expression profiles will cluster together in the PCA plot. This analysis, using the whole probe set on the chip, resulted in a clear segregation of the 12 replicated samples into three clusters, corresponding to IVF, IVC, and in vivo (Fig. 3B).

\section{Analysis of classes of genes and gene pathways altered in the experimental groups IVF versus in vivo control}

Results of gene ontology in IVF embryos when compared with control embryos are summarized in Table 3. IVF embryos show an up-regulation of cell cycle, apoptosis, RNA metabolism and phosphoenolpyruvate-dependent sugar phosphotransferase system gene categories. Embryonic development genes and morphogenesis genes were down-regulated and this can explain a reduced

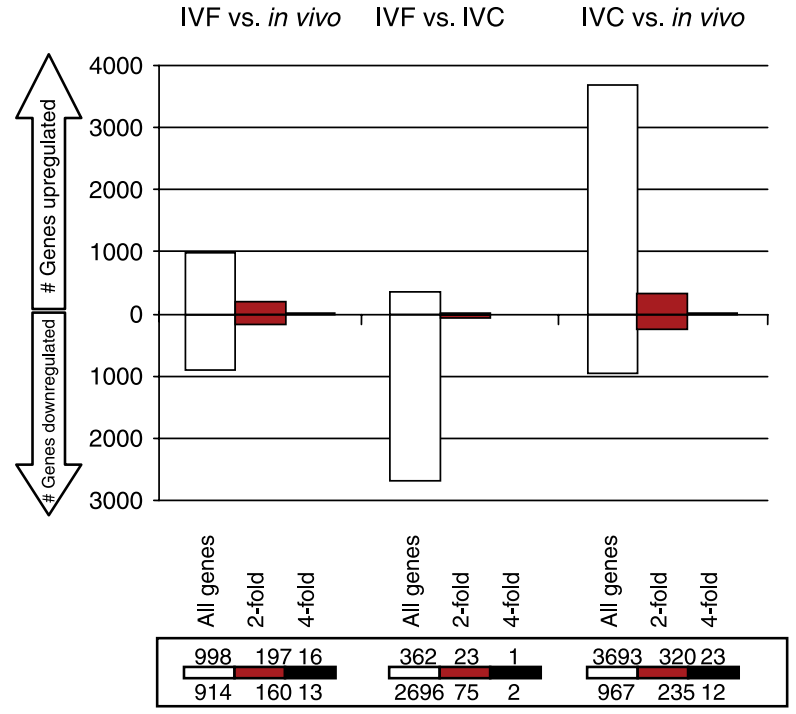

Figure 2 Number of transcripts differentially regulated according to the fertilization method. The number of genes either down- or up-regulated is the number that is statistically different after direct comparison of the described experimental group. The exact number of genes is specified in the inferior portion of the graph.

performance of the IVF embryos. Genes involved in heart development, forebrain development, bone mineralization, and segmentation are among the family of morphogenetic genes mis-expressed. Additional classes of down-regulated genes consist of amino acid transport and metabolism genes, fat cell differentiation, fluid transport, and genes involved in intracellular signaling cascade. Of particular interest is the down-regulation of pathways important in early development, such as the cell cycle, apoptosis, and Wnt signaling pathways.

\section{IVF versus IVC}

This comparison allows us to observe and isolate the effect of the conditions at fertilization (in vitro versus in vivo), since, the culture conditions from the zygote stage to the blastocyst stage were exactly the same in the two groups.

Surprisingly, IVF embryos developed few hours faster from the two-cell stage to the blastocyst stage than IVC embryos. On the other hand, IVF embryos had a reduced number of TB cells $(P<0.01)$ and an equal number of ICM cells. The average area of the ICM cell nucleus was larger in the IVF group.

Gene expression was remarkably different: more than 3000 genes were statistically different between the two groups (3058 transcripts, Fig. 2). Eighty-eight percent (2696/3058) of the IVF transcripts were down-regulated; however, the intensity of change was small and only 98 genes $(0.03 \%, 98 / 3058)$ were modified more than twofold.

Analysis of common processes and gene pathways revealed that overall IVF embryos displayed up-regulation of protein biosynthesis and protein amino acid 
A
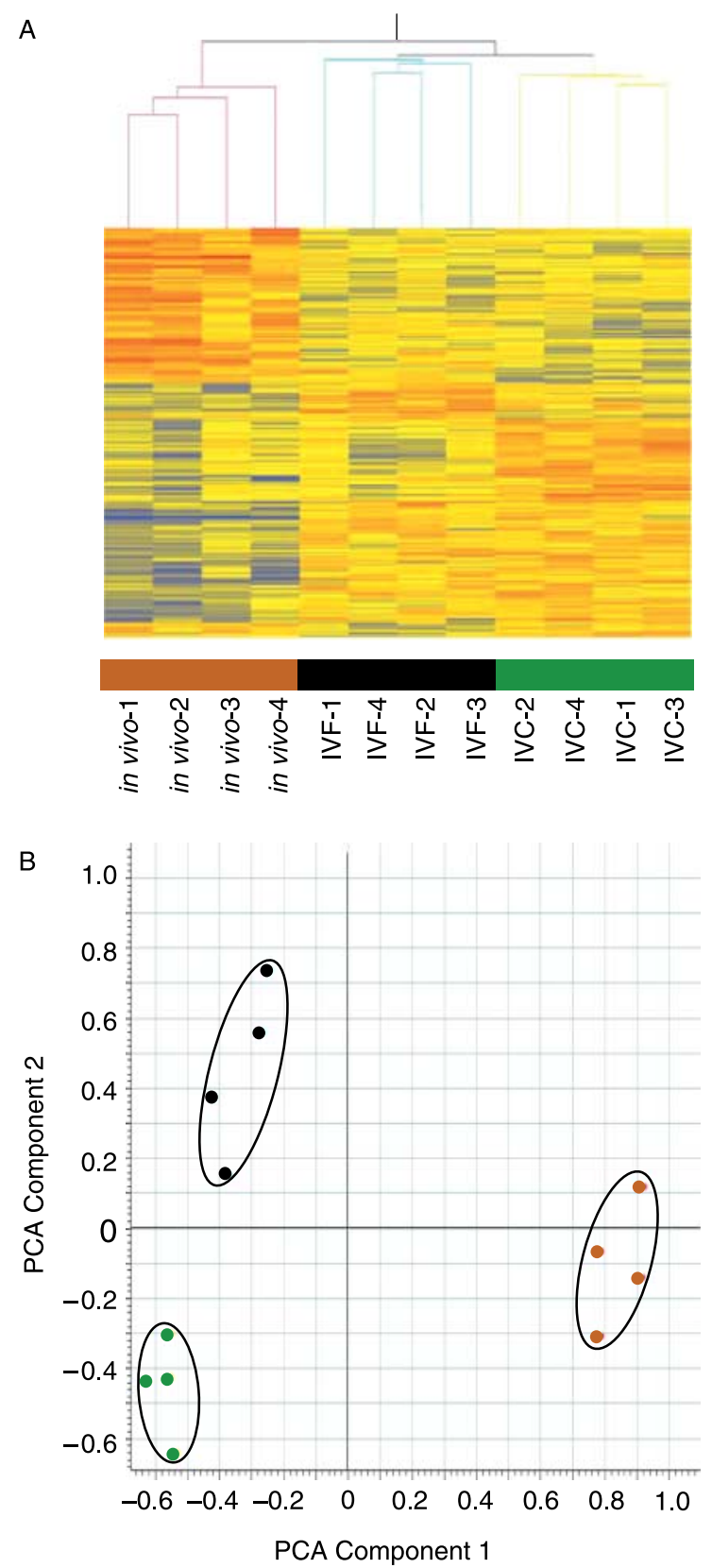

Figure 3 Hierarchical clustering and PCA analysis. (A) Hierarchical clustering analysis of all samples from different developmental stages or treatments. Unsupervised clustering in GeneSpring was used to analyze similarities among replicate samples across all treatment groups tested. Colors correspond to relative RNA abundance for the transcripts detected; each is represented by a horizontal bar in the heat-map. Red indicates up-regulation, blue denotes down-regulation and yellow no change in expression. Note the appropriate clustering of data from the four samples derived from IVF-generated embryos (brown lines/bars), the four samples from IVC embryos (green lines) and the four samples derived from embryos that developed in vivo (black lines/bars). In vivo, IVF and IVC refer to blastocysts developed in vivo, cultured in vitro after fertilization in vitro and cultured in vitro after fertilization in vivo, respectively. (B) Principal component analysis of gene expression of all groups as in the hierarchical clustering analysis. glycosylation genes and down-regulation of steroid and lipid metabolism and transport genes (Table 4). It is particularly interesting to note that heart development genes and genes involved in axon guidance are downregulated in the IVF group.

\section{Discussion}

This study establishes that IVF has a discrete and distinctive effect on the phenotype and gene expression pattern of mouse preimplantation embryos. The differences between IVF and in vivo embryos are quite remarkable. The most unanticipated findings are the net reduction of TE cells and increase in sizes of ICM cell nuclei in the IVF group when compared with the in vivo control. The gene expression results paralleled the morphologic results and showed that genes involved in development, cell division, and apoptosis were misregulated following IVF. Interestingly, although we expected the IVF and IVC groups to be similar, we found remarkable differences between these two groups both in gene expression and morphology.

The increase in size of the nucleus in the IVF embryos was surprising and unexpected and its significance is unclear. Because we only stained the nucleus and did not visualize the cytoplasm, we cannot infer that the nucleocytoplasmic ratio is altered. Only ultrastructural examination will help clarify the issue. Other authors have observed changes in the morphology of preimplantation embryos after culture. Crosier et al. (2001) analyzed the ultrastructural morphometry of bovine blastocysts produced in vivo or in vitro using three different culture media and noted that in vitro blastocysts had a smaller nucleocytoplasmic ratio. They associated this finding with reduced quality of in vitro embryos. However, Petzoldt \& Muggleton-Harris (1987) manipulated the nucleocytoplasmic ratio of mouse preimplantation embryos by removing or injecting cytoplasm and concluded that gene stage specific gene activity was independent of this ratio.

The different morphology in cultured embryos suggest that a number of fundamental processes are altered by the culture condition; i.e., cell proliferation, apoptosis, and/or allocation of cells to the ICM or TE lineages are different depending upon whether fertilization occurs in vivo or in vitro. Together, these findings suggest that blastocysts produced by IVF may be at a significant developmental disadvantage when compared with those that develop in vivo. In agreement with our findings, Trounson \& Bongso (1996) reported that human IVF embryos often show abnormal or delayed cell division. Hence, we postulated that the observed embryo development and number of cells present in the ICM and TE layers might significantly be associated with differences in gene expression in IVF embryos.

Indeed, the gene expression results confirm the morphologic studies. The IVF embryos have a very 
Table 3 Gene ontology of family of genes statistically different between in vitro fertilization (IVF) and in vivo generated control blastocysts.

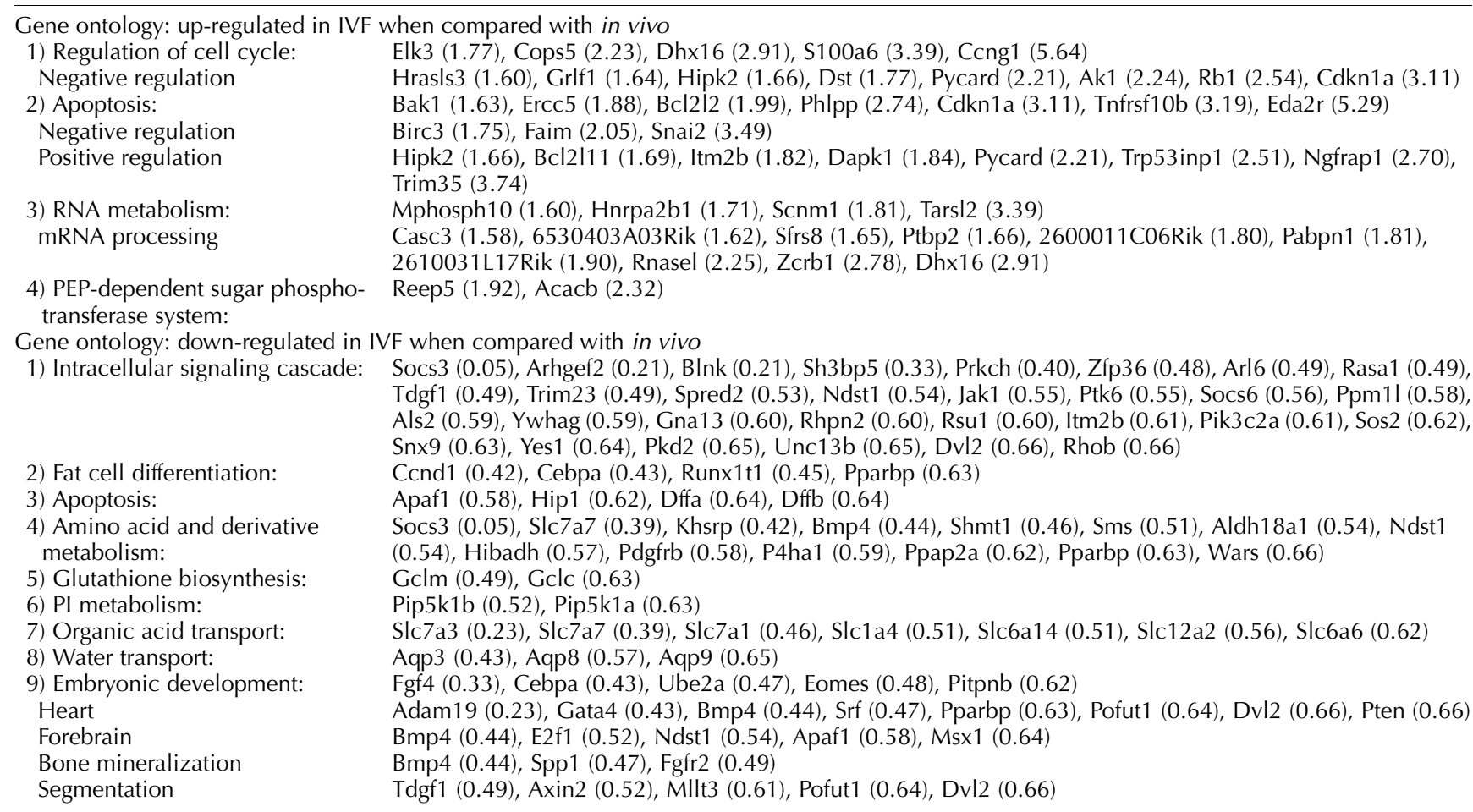

distinct fingerprint when compared with embryos fertilized in vivo, as shown by the PCA and the unsupervised hierarchical analysis (Fig. 3). Overall, the IVF embryos have an increased alteration (both up and down-regulation) in the expression of apoptotic genes. This could explain the reduced number of TE cells. The down-regulation of several genes involved in differentiation (fat cell differentiation, heart and forebrain development, bone mineralization and segmentation) are provocative and open the possibility that important alterations of fundamental process are taking place. In fact, knock out animals for several of these genes have very specific anomalous phenotypes. For example, Srf (Arsenian et al. 1998, Li et al. 2005a, 2005b, Niu et al. 2005) and Eomes (Russ et al. 2000) knock out mice exhibit abnormal gastrulation and Ube2a mutation has been shown to reduce the embryonic growth (Roest et al. 2004). In addition, mutation in the Eomes gene, is shown to cause abnormal trophoblast development (Russ et al. 2000), a particular important findings in our study, given the reduced number of TE cells in the IVF blastocysts. In addition, Gata4 is able to induce visceral trophectoderm from ES cells (Fujikura et al. 2002).

Whether differences in gene expression will persist throughout embryonic development is not known. However, a group of genes involved in cardiac development (Gata4 (Kuo et al. 1997, Tevosian et al. 2002), Adam19 (Kurohara et al. 2004), Fgf4, and Fgfr2 (Feldman et al. 1995, Wang et al. 2005), and Bmp4 (Liu et al. 2004, Zakin \& De Robertis 2004) are down-

Table 4 Gene ontology of family of genes statistically different between in vitro fertilization (IVF) and in vitro culture (IVC) generated blastocysts.

\begin{tabular}{|c|c|}
\hline \multicolumn{2}{|l|}{ Gene ontology up-regulated genes in IVF when compared with IVC } \\
\hline 1) Protein targeting: $P=0.0063$ & $\begin{array}{l}\text { Dmgdh (1.72), Ormdl1 (1.72), 1700034H14Rik (1.64), Al317395 (1.85) } \\
\text { Kpna4 (1.92) }\end{array}$ \\
\hline 2) Biosynthesis: $P=0.0079$ & Nos1 (1.59), Sc4mol (1.61), Cds2 (1.72) \\
\hline Protein biosynthesis & $\begin{array}{l}\text { Nmt2 (1.52), Eif2c1 (1.72), Impact (1.75), Mrps21 (1.82), Mtrf1I (2.00), } \\
\text { Ptrh1 (2.00) }\end{array}$ \\
\hline Protein amino acid glycosylation & $\begin{array}{l}\text { Nipsnap1 (1.52), Ogt (1.52), Sfxn1 (1.52), Timm22 (1.56), Edem1 (1.61) } \\
\text { Mrps21 (1.82), St3gal3 (1.82) }\end{array}$ \\
\hline \multicolumn{2}{|l|}{ Gene ontology: down-regulated in IVF when compared with IVC } \\
\hline 1) Enzyme linked receptor protein signaling pathway: $P=0.0080$ & $\begin{array}{l}\text { Prkca (1.55), Acvr1 (1.65), Ptpro (1.73), Htra1 (1.82), Nrp1 (1.84), Epha } \\
\text { (1.88), Ltbp1 (1.89), Ephb2 (1.92) }\end{array}$ \\
\hline cAMP-mediated signalling & Ptger3 (1.70), Oprm1 (1.96), Rims2 (2.00), Gnal (2.23) \\
\hline 2) Steroid metabolism: $P=0.0070$ & Pbx1 (1.64), Akr1c21 (1.68) \\
\hline Steroid/lipid transport & Osbp2 (1.62), Abca1 (1.65), Apoa1 (1.75), Vldlr (2.07) \\
\hline 3) Heart development: $P=0.0094$ & Acvr1 (1.65), Tgfb2 (1.76), Nrp1 (1.84), Nrp2 (2.08), Myocd (2.20) \\
\hline
\end{tabular}


regulated, and Cebpa knock outs show abnormalities in adipogenesis, hematopoiesis, hepatic, and lung morphology and premature death (Flodby et al. 1996, Zhang et al. 2004). It is possible that these changes are temporary and disappear, but could persist and affect long term development, extending the Barker hypothesis (Barker 1998) to the preimplantation period.

IVC and IVF embryos were more different from each other than was expected. For example, there was a progressive and significant decrease in TE cell number as the time in culture and degree of manipulation increased (in vivo $>$ IVC $>$ IVF). The number of ICM cells was reduced after IVC, but more so in the IVC group. This indicates a specific effect on the TE cells either in terms of proliferation or survival in the IVF embryos. Interestingly, a statistically higher number of IVF embryos developed from the two-cell stage to the blastocyst stage when compared with IVC embryos. In agreement to these findings, more genes were statistically abnormal after IVC (IVC versus in vivo: 4660 different genes) than after IVF (IVF versus in vivo comparison: 1912 different genes). These latter findings were surprising and contrary to our expectation. In fact, it is well known that embryos develop more slowly in culture when compared with the in vivo counterpart. Additional time spent in culture, and in particular the first $24 \mathrm{~h}$ of development, would have been expected to slow the development even further. Our findings, however, suggest that a change in environment (like the one present in IVC embryos fertilized in vivo and then cultured in vitro) appear to be more stressful to the embryos; one could speculate that embryos benefit from being in the same environment from fertilization onward, no matter how artificial the initial environment is. A change in environment represents a stressful shock, requiring time to adapt to the new condition. The timing of this particular stressor is important in determining its effect. It would be interesting to perform complementary experiments (IVF and transfer of zygotes in the fallopian tubes) to test this hypothesis.

Direct comparison of differentially expressed genes in IVF versus IVC reveals that several abnormally regulated IVF genes are involved in brain development. Mutation of Nos1 has been shown to increase neurogenesis and mutation of Nrp1, Nrp2 (down-regulated 1.8 and 2 times respectively) has been reported to induce abnormal brain development (Gu et al. 2003) and abnormal neurogenesis and axon guidance (Chen et al. 2000). Tgfb2, in addition to causing abnormal brain development, is associated with abnormal cardiovascular, respiratory, and reproductive morphology. This indicates that although the IVF procedure shows fewer alterations in the level of specific gene expression when compared with IVC, some of the important morphogenic genes are altered in the IVF embryos.

The comparison of IVC versus in vivo has been the subject of two prior publications (Rinaudo \& Schultz
2004, Rinaudo et al. 2006). Overall, our new results confirm our past experiments. There are some notable differences in number of genes statistically different (4660 genes in the present comparison IVC versus in vivo in $20 \%$ oxygen versus 1159 genes in the past comparison), but the classes of genes altered are the same. The differences in gene number are likely due to the differences in laboratory environment, RNA amplification technique (double amplification protocol versus linear amplification using the NuGen protocol used in the present experiments) and the gene chip used in this study. In fact, in the prior experiments, we used the Affymetrix MOE 430A chip, containing only 22000 transcripts. The present set of experiments used the NuGen small sample amplification protocol because this protocol is robust and requires a reduced amount of starting RNA. In particular, the NuGen linear amplification protocol conserves the correct ratio between mRNAs that are expressed at different levels and does not distort transcript abundance. (Goff et al. 2004, Barker et al. 2005).

Our study is the first one to offer a complete overview of the changes in the global pattern of gene expression in IVF versus in vivo generated mouse embryos and completes our prior findings (Rinaudo \& Schultz 2004, Rinaudo et al. 2006).

Other authors have analyzed the effect of the fertilization method on mammalian embryos and their findings are complementary to ours. Whitworth et al. (2005) analyzed the differences between IVF and in vivo embryos in the porcine model by using a tissue-specific 19968 spot cDNA microarray. Interestingly, cluster analysis revealed that IVF and control embryos were different at the four-cell stage, but they did not separate in independent branches at the blastocyst stage, indicating a less dramatic effect of culture on embryos at this latter stage. One hypothesis advanced by the authors is that poor IVF quality embryos were removed at (or after) the four-cell stage therefore allowing for a more uniform 'healthy' blastocyst population. This could be a species- specific phenomenon. However, the two classes of embryos had significant differences: there were 1696 differentially expressed genes between the two groups and similar gene pathways were modified; for example, RNA metabolism and ribonucleoprotein complex were up-regulated in IVF embryos, a finding consistent with our observation.

Global pattern of gene expression in in vitro versus in vivo produced embryos was also analyzed by Corcoran et al. (2006) in bovine embryos. They found 384 genes differentially regulated in IVF embryos. There was a general down-regulation of genes involved in transcription and translation, as well as genes involved in cellular metabolism.

One potential limitation of our experiments is the choice of WM, a medium that is known to be suboptimal. In fact, we have shown in the past that 
WM results in higher number of mis-regulated genes than KSOM medium with amino acids (Rinaudo \& Schultz 2004) and that a high oxygen concentration (20\% or atmospheric oxygen) has an additional and synergistic negative effect on the gene expression pattern (Rinaudo et al. 2006). However, we specifically decided to use WM to stress the system and therefore, potentially magnify the effects of a different method of fertilization. In this fashion, we wanted to glimpse the potential mechanism by which the method of fertilization affects embryo health. Future experiments will be conducted to assess the effect of an improved media and different methods of fertilization on the global pattern of gene expression.

Another important aspect to note is that we compared mRNA abundances between blastocysts that appeared identical at the time of the selection (at $60 \times$ using a stereomicroscope), but when later examined for cell numbers, had different quantity of cells. Therefore, the results described could simply be explained by the fact that the blastocysts were at slightly different stage of development. We exclude this possibility, because we were careful to select only late cavitating blastocysts of similar morphology, as we have done in our prior work (Rinaudo \& Schultz 2004, Rinaudo et al. 2006). In addition, the cell number differences only involve the TE cells, while the number of ICM cells is similar in all three groups. For example, the IVC group and IVF group have a comparable number of ICM cells (12 and 13 respectively), but a statistically different number of TE cells (40 and 34 respectively). If the blastocysts were truly at different stages of development we would expect the ICM number to be lower in the IVF group. Indeed, we think that all the groups are in same stage of differentiation and the difference in cell numbers are secondary to increase incidence of apoptosis or decrease in cell proliferation, as confirmed by our microarray data. Other authors (Devreker \& Hardy 1997) have documented a significant change in cell number, mitotic, and apoptotic index in blastocysts cultured in different media.

In conclusion, the in vitro produced mouse embryos in the specific culture conditions used are at a disadvantage when compared with in vivo embryos. It remains to be investigated if these changes are only temporary or are associated with long-term consequences. For example, it is unclear if the down-regulation of the embryonic development genes is associated with delayed patterning in the cultured embryos. Presence of a long-term effect would be in agreement with the Barker hypothesis that holds that in utero events are associated with long lasting predisposition to disease (Barker 1998). Another question to be elucidated is whether changes reported in one species might be present in another species. At present, for example, it appears that bovine embryos are affected by the large offspring syndrome (Niemann \& Wrenzycki 2000), while human embryos are not (Olivennes et al.
2002). More studies need to be performed to evaluate what is the effect of other forms of artificial fertilization (i.e. ICSI) on embryo development.

\section{Acknowledgements}

F D has been supported by a grant from Fondo A Griffini e J Miglierina awarded by Amministrazione Provinciale di Varese, Italy in 2006. The authors declare that there is no conflict of interest that would prejudice the impartiality of this scientific work. This work has been partially supported by an ASRM/Serono grant, a Bechtel grant and a WRHR grant to P R. The authors wished to thank Dr Emin Maltepe for his valuable suggestions.

\section{References}

Anthony SBS, Dorrepaal C, Lindner K, Braat D \& den Ouden A 2002 Congenital malformations in 4224 children conceived after IVF. Human Reproduction 17 2089-2095.

Arsenian S, Weinhold B, Oelgeschlager M, Ruther U \& Nordheim A 1998 Serum response factor is essential for mesoderm formation during mouse embryogenesis. EMBO Journal 17 6289-6299.

Ashburner M, Ball CA, Blake JA, Botstein D, Butler H, Cherry JM, Davis AP, Dolinski K, Dwight SS, Eppig JT et al. 2000 Gene ontology: tool for the unification of biology. The Gene Ontology Consortium. Nature Genetics 25 25-29.

Barker DJ 1998 Mothers, Babies and Health in Later Life, 2 Churchill Livingstone: Glasgow.

Barker CS, Griffin C, Dolganov GM, Hanspers K, Yang JY \& Erle DJ 2005 Increased DNA microarray hybridization specificity using sscDNA targets. BMC Genomics 657.

Baum M, Bielau S, Rittner N, Schmid K, Eggelbusch K, Dahms M, Schlauersbach A, Tahedl H, Beier M, Guimil R et al. 2003 Validation of a novel, fully integrated and flexible microarray benchtop facility for gene expression profiling. Nucleic Acids Research 31 e151.

Chen H, Bagri A, Zupicich JA, Zou Y, Stoeckli E, Pleasure SJ, Lauenstein DH, Skarnes WC, Cheodotal A \& Tessier-Lavigne M 2000 Neurophilin-2 regulates the development of selective cranial and sensory nerves and hippocampal mossy fiber projections. Neuron 25 43-56.

Corcoran D, Fair T, Park S, Rizos D, Patel OV, Smith GW, Coussens PM, Ireland JJ, Boland MP, Evans AC et al. 2006 Suppressed expression of genes involved in transcription and translation in in vitro compared with in vivo cultured bovine embryos. Reproduction 131 651-660.

Cox GF, Burger J, Lip V, Mau UA, Sperling K, Wu BL \& Horsthemke B 2002 Intracytoplasmic sperm injection may increase the risk of imprinting defects. American Journal of Human Genetics 71 162-164.

Crosier AE, Farin PW, Dykstra MJ, Alexander JE \& Farin CE 2001 Ultrastructural morphometry of bovine blastocysts produced in vivo or in vitro. Biology of Reproduction 64 1375-1385.

DeBaun MR, Niemitz EL \& Feinberg AP 2003 Association of in vitro fertilization with Beckwith-Wiedemann syndrome and epigenetic alterations of LIT1 and H19. American Journal of Human Genetics 72 156-160.

Devreker F \& Hardy K 1997 Effects of glutamine and taurine on preimplantation development and cleavage of mouse embryos in vitro. Biology of Reproduction 57 921-928.

Ecker DJ, Stein P, Xu Z, Williams CJ, Kopf GS, Bilker WB, Abel T \& Schultz RM 2004 Long-term effects of culture of preimplantation mouse embryos on behavior. PNAS 101 1595-1600.

Feldman B, Poueymirou W, Papaioannou VE, DeChiara TM \& Goldfarb M 1995 Requirement of FGF-4 for postimplantation mouse development. Science 267 246-249. 
Fernandez-Gonzalez R, Moreira P, Bilbao A, Jimenez A, PerezCrespo M, Ramirez MA, Rodriguez De Fonseca F, Pintado B \& Gutierrez-Adan A 2004 Long-term effect of in vitro culture of mouse embryos with serum on mRNA expression of imprinting genes, development, and behavior. PNAS 101 5880-5885.

Flodby P, Barlow C, Kylefjord H, Ahrlund-Richter L \& Xanthopoulos KG 1996 Increased hepatic cell proliferation and lung abnormalities in mice deficient in CCAAT/enhancer binding protein alpha. Journal of Biological Chemistry 271 24753-24760.

Fujikura J, Yamato E, Yonemura S, Hosoda K, Masui S, Nakao K, Miyazaki Ji J \& Niwa H 2002 Differentiation of embryonic stem cells is induced by GATA factors. Genes and Development 16 784-789.

Gu C, Rodriguez ER, Reimert DV, Shu T, Fritzsch B, Richards LJ, Kolodkin AL \& Ginty DD 2003 Neurophilin 1 conveys semaphorin and VEGF signating during neural and cardiovascular development. Developmental Cell 5 45-57.

Goff LA, Bowers J, Schwalm J, Howerton K, Getts RC \& Hart RP 2004 Evaluation of sense-strand mRNA amplification by comparative quantitative PCR. BMC Genomics 576.

Hansen M, Kurinczuk JJ, Bower C \& Webb S 2002 The risk of major birth defects after intracytoplasmic sperm injection and in vitro fertilization. New England Journal of Medicine 346 725-730.

Irizarry RA, Hobbs B, Collin F, Beazer-Barclay YD, Antonellis KJ, Scherf U \& Speed TP 2003 Exploration, normalization, and summaries of high density oligonucleotide array probe level data. Biostatistics 4 249-264.

Joliffe IT \& Morgan BJ 1992 Principal component analysis and exploratory factor analysis. Statistical Methods in Medical Research 1 69-95.

Kovalevsky G, Rinaudo P \& Coutifaris C 2003 Do assisted reproductive technologies cause adverse fetal outcomes? Fertility and Sterility $\mathbf{7 9}$ 1270-1272.

Kuo CT, Morrisey EE, Anandappa R, Sigrist K, Lu MM, Parmacek MS, Soudais C \& Leiden JM 1997 GATA4 transcription factor is required for ventral morphogenesis and heart tube formation. Genes and Development 11 1048-1060.

Kurohara K, Komatsu K, Kurisaki T, Masuda A, Irie N, Asano M, Sudo K, Nabeshima Y, Iwakura Y \& Sehara-Fujisawa A 2004 Essential roles of meltrin beta (ADAM19) in heart development. Developmental Biology 267 14-28.

Li S, Czubryt MP, McAnally J, Bassel-Duby R, Richardson JA, Wiebel FF, Nordheim A \& Olson EN 2005a Requirement for serum response factor for skeletal muscle growth and maturation revealed by tissuespecific gene deletion in mice. PNAS 102 1082-1087.

Li T, Vu TH, Ulaner GA, Littman E, Ling JQ, Chen HL, Hu JF, Behr B, Giudice L \& Hoffman AR 2005b IVF results in de novo DNA methylation and histone methylation at an Igf2-H19 imprinting epigenetic switch. Molecular Human Reproduction 11 631-640.

Liu W, Selever J, Wang D, Lu MF, Moses KA, Schwartz RJ \& Martin JF 2004 Bmp4 signaling is required for outflow-tract septation and branchial-arch artery remodeling. PNAS 101 4489-4494.

Niemann H \& Wrenzycki C 2000 Alterations of expression of developmentally important genes in preimplantation bovine embryos by in vitro culture conditions: implications for subsequent development. Theriogenology 53 21-34.

Niu Z, Yu W, Zhang SX, Barron M, Belaguli NS, Schneider MD, Parmacek M, Nordheim A \& Schwartz RJ 2005 Conditional mutagenesis of the murine serum response factor gene blocks cardiogenesis and the transcription of downstream gene targets. Journal of Biological Chemistry 280 32531-32538.

Olivennes F, Fanchin R, Ledee N, Righini C, Kadoch IJ \& Frydman R 2002 Perinatal outcome and developmental studies on children born after IVF. Human Reproduction Update 8 117-128.

Petzoldt U \& Muggleton-Harris A 1987 The effect of the nucleocytoplasmic ratio on protein synthesis and expression of a stage-specific antigen in early cleaving mouse embryos. Development 99 $481-491$.
Rinaudo P \& Schultz RM 2004 Effects of embryo culture on global pattern of gene expression in preimplantation mouse embryos. Reproduction 128 301-311.

Rinaudo PF, Giritharan G, Talbi S, Dobson AT \& Schultz RM 2006 Effects of oxygen tension on gene expression in preimplantation mouse embryos. Fertility and Sterility $\mathbf{8 6} 1252-1265$.

Roest HP, Baarends WM, de Wit J, van Klaveren JW, Wassenaar E, Hoogerbrugge JW, van Cappellen WA, Hoeijmakers JH \& Grootegoed JA 2004 The ubiquitin-conjugating DNA repair enzyme HR6A is a maternal factor essential for early embryonic development in mice. Molecular and Cellular Biology 24 5485-5495.

Russ AP, Wattler S, Colledge WH, Aparicio SA, Carlton MB, Pearce JJ, Barton SC, Surani MA, Ryan K, Nehls MC et al. 2000 Eomesodermin is required for mouse trophoblast development and mesoderm formation. Nature 404 95-99.

Schieve LA, Meikle SF, Ferre C, Peterson HB, Jeng G \& Wilcox LS 2002 Low and very low birth weight in infants conceived with use of assisted reproductive technology. New England Journal of Medicine 346 731-737.

Stromberg BDG, Ericson A, Finnstrom O, Koster M \& Stjernqvist $\mathrm{K}$ 2002 Neurological sequelae in children born after in-vitro fertilization: a population-based study. Lancet 359 461-465.

Tevosian SG, Albrecht KH, Crispino JD, Fujiwara Y, Eicher EM \& Orkin SH 2002 Gonadal differentiation, sex determination and normal Sry expression in mice require direct interaction between transcription partners GATA4 and FOG2. Development 129 4627-4634.

Thouas GA, Korfiatis NA, French AJ, Jones GM \& Trounson AO 2001 Simplified technique for differential staining of inner cell mass and trophectoderm cells of mouse and bovine blastocysts. Reproductive Biomedicine Online 3 25-29.

Trounson A \& Bongso A 1996 Fertilization and development in humans. Current Topics in Developmental Biology 32 59-101.

Wang Y, Xiao R, Yang F, Karim BO, lacovelli AJ, Cai J, Lerner CP, Richtsmeier JT, Leszl JM, Hill CA et al. 2005 Abnormalities in cartilage and bone development in the Apert syndrome FGFR2(+ /S252W) mouse. Development 132 3537-3548.

Whitworth KM, Agca C, Kim JG, Patel RV, Springer GK, Bivens NJ, Forrester LJ, Mathialagan N, Green JA \& Prather RS 2005 Transcriptional profiling of pig embryogenesis by using a $15-\mathrm{K}$ member unigene set specific for pig reproductive tissues and embryos. Biology of Reproduction 72 1437-1451.

Zakin L \& De Robertis EM 2004 Inactivation of mouse Twisted gastrulation reveals its role in promoting Bmp4 activity during forebrain development. Development 131 413-424.

Zeng F \& Schultz RM 2005 RNA transcript profiling during zygotic gene activation in the preimplantation mouse embryo. Developmental Biology 283 40-57.

Zhang P, Iwasaki-Arai J, Iwasaki H, Fenyus ML, Dayaram T, Owens BM, Shigematsu H, Levantini E, Huettner CS, Lekstrom-Himes JA et al. 2004 Enhancement of hematopoietic stem cell repopulating capacity and self-renewal in the absence of the transcription factor C/EBP alpha. Immunity 21 853-863.

Zhang B, Kirov S \& Snoddy J 2005 WebGestalt: an integrated system for exploring gene sets in various biological contexts. Nucleic Acids Research 33 W741-W748.

Received 4 October 2006

First decision 26 October 2006

Revised manuscript received 18 March 2007

Accepted 22 March 2007 